\title{
Measuring the Efficacy of Leaders to Assess Information and Make Decisions in a Crisis: The C- LEAD Scale.
}

\section{Citation}

Hadley, Constance Noonan, Todd L. Pittinsky, S. Amy Sommer, and Weichun Zhu. 2009.

Measuring the Efficacy of Leaders to Assess Information and Make Decisions in a Crisis: The CLEAD Scale. HKS Faculty Research Working Paper Series RWP09-021, John F. Kennedy School of Government, Harvard University.

\section{Published Version}

http://web.hks.harvard.edu/publications/workingpapers/citation.aspx?Publd=6713

\section{Permanent link}

http://nrs.harvard.edu/urn-3:HUL.InstRepos:4448991

\section{Terms of Use}

This article was downloaded from Harvard University's DASH repository, and is made available under the terms and conditions applicable to Other Posted Material, as set forth at http:// nrs.harvard.edu/urn-3:HUL.InstRepos:dash.current.terms-of-use\#LAA

\section{Share Your Story}

The Harvard community has made this article openly available.

Please share how this access benefits you. Submit a story.

Accessibility 


\author{
Faculty Research Working Papers Series
}

\title{
Measuring the Efficacy of Leaders to Assess Information and Make Decisions in a Crisis: The C-LEAD Scale
}

\author{
Constance Noonan Hadley, Todd L. Pittinsky \\ John F. Kennedy School of Government - Harvard University
}

S. Amy Sommer

Department of Psychology- Harvard University

Weichun Zhu

Claremont McKenna College

July 2009

RWP09-021

The views expressed in the HKS Faculty Research Working Paper Series are those of the author(s) and do not necessarily reflect those of the John F. Kennedy School of Government or of Harvard University. Faculty Research Working Papers have not undergone formal review and approval. Such papers are included in this series to elicit feedback and to encourage debate on important public policy challenges. Copyright belongs to the author(s). Papers may be downloaded for personal use only. 
Measuring the Efficacy of Leaders to Assess Information and Make Decisions in a Crisis:

The C-LEAD Scale

\author{
Constance Noonan Hadley, Todd L. Pittinsky, and S.Amy Sommer \\ Harvard University \\ Weichun Zhu \\ Claremont McKenna College
}

Corresponding author: Constance Noonan Hadley, Harvard Kennedy School, Harvard University, 79 John F. Kennedy, Street, Cambridge, MA 02138, Tel.: (617) 480-7660; Fax: (617) 496-3337. E-mail: Connie_hadley@hks.harvard.edu

Authors' note: This research has been funded by the Robert Wood Johnson Foundation, the Centers for Disease Control and Prevention (CDC), and the CDC Foundation. The authors thank Brian Welle, H’Sien Hayward, Seth A. Rosenthal, Erika James, R. Matthew Montoya, Laura Maruskin, Laura Bacon, Stefanie Simon, Donna Kalikow, Howard Koh, and three anonymous reviewers at The Leadership Quarterly for helpful input and comments on earlier versions of this manuscript.

Citation: Hadley, C.N., Pittinsky, T. L., Sommer, S. A., \& Zhu, W. (2009). Measuring the efficacy of leaders to assess information and make decisions in a crisis: The C-LEAD Scale. Unpublished manuscript. 
Measuring the Efficacy of Leaders to Assess Information and Make Decisions in a Crisis: The C-LEAD Scale

\begin{abstract}
Based on literature and expert interviews, we developed the Crisis Leader Efficacy in Assessing and Deciding scale (C-LEAD) to capture the efficacy of leaders to assess information and make decisions in a public health and safety crisis. In Studies 1 and 2, we find that C-LEAD predicts decision-making difficulty and confidence in a crisis better than a measure of general leadership efficacy. In Study 3, C-LEAD predicts greater motivation to lead in a crisis, more crisis leader role-taking, and more accurate performance while in a crisis leader role. These findings support the scale's construct validity and broaden our theoretical understanding of the nature of crisis leader efficacy.
\end{abstract}

Keywords: Crisis leadership, public health and safety, information assessment, decision making 
Measuring the efficacy of leaders to assess information and make decisions in a crisis:

The C-LEAD Scale

\section{Introduction}

During any public health and safety crisis, whether it is an airplane crash, hurricane, infectious disease pandemic, or terrorist act, there is a tremendous need for effective leadership. In particular, good decisions must be made quickly, despite the uncertainty, time pressure, and high stakes associated with such a crisis (Pearson \& Clair, 1998). Furthermore, information must be assessed and decisions must be made among all those involved in the response effort, from the senior federal officials directing from a command center, to the local personnel treating the affected in the field (Gorge, 2006; Mitroff, 2004). Despite the significant and widespread need for capable leaders in a crisis, however, prior research and theory has not provided a tool with which to assess the efficacy of individuals to perform these essential tasks of information assessment and decision making while under crisis circumstances. The current research aims to develop and validate such a tool so that we may increase our ability to recognize, train, and leverage those current and potential leaders who will be effective decision-makers in a crisis.

In general, research on crisis tends to be conceptual (e.g., Mitroff \& Alpaslan, 2003; Sweeny, 2008) or based on unique case studies of singular events, such as the Challenger explosion (Vaughan, 1996) or the Iraq war (Bateman, 2008), although a few researchers have conducted crisis field studies (e.g., Pillai \& Meindl, 1998) and laboratory experiments (e.g., Halverson, Murphy, \& Riggio, 2004; Hunt, Boal, \& Dodge, 1999). This literature has been useful in providing broad frameworks of crisis management with an emphasis on the role of transformational and charismatic leadership approaches (e.g., Halverson, et al., 2004; Pearson \& 
Clair, 1998). However, there is very little research about the specifics of how leaders effectively respond to a crisis (e.g., Sweeny, 2008; Wooten \& James, 2008) and how the capabilities of leaders can be assessed in advance of a crisis occurring (Schoenberg, 2005). Such knowledge is necessary to develop a more systematic and comprehensive understanding of the nature of crisis leadership and facilitate the development of capability-building interventions before crisis events occur. In this paper, we present an empirically-derived measure of effective crisis leadership, the Crisis Leader Efficacy in Assessing and Deciding scale (C-LEAD). We focus on a leader's selfefficacy to perform two core aspects of crisis response: assess information and make decisions (Boin, 't Hart, Stern, \& Sundelius, 2005; Coombs, 2005; Wooten \& James, 2008).

Self -efficacy is a personal judgment of "how well one can execute courses of action required to deal with prospective situations" (Bandura, 1982, p. 122) and is related to actual performance in a number of different contexts (Hannah, Avolio, Luthans, \& Harms, 2008; Saks, 1995; Stajkovic \& Luthans, 1998). In empirical tests using C-LEAD, we explore the nature of crisis leader efficacy and its relation to other constructs. Overall, the current research provides a new means of assessing an important aspect of crisis leadership and lays the groundwork for additional research, theory, and practice in the area.

\subsection{Crisis leadership in public health and safety crises}

As we have seen throughout history, the consequences of ineffective leadership in a crisis can be both costly and deadly (Mitroff, 2004; Tichy \& Bennis, 2007). Our research focuses on public health and safety crises, which are defined as a low-probability, high-impact events that threaten the security and well-being of the public (adapted from Pearson \& Clair, 1998). We focus on public health and safety crises because such crises have received less attention in leadership research than other forms of crises, such as corporate scandals and financial crises 
(e.g., Fowler, Kling, \& Larson, 2007; Pang, Cropp, \& Cameron, 2006), and yet they carry enormous significance for human life and safety. As is true for most forms of crisis, public health and safety crises involve ambiguity in terms of cause, effect and means of resolution (Dutton, 1986; Pearson \& Clair, 1998; Quarantelli, 1988), as well as urgency to resolve the situation as quickly as possible (Billings, Milburn, \& Schaalman, 1980; Quarantelli, 1988). In addition, the stakes involved in public health and safety crises are extraordinarily high: the lives and welfare of human beings are at risk (Mitroff, 2004; Shrivastava, 1993). Given the scope and magnitude of such crises, leadership challenges can be extreme (Mitroff, 2004).

In a crisis, leaders are called upon to perform many actions, including clarifying the situation to external audiences, conveying hope and resoluteness to the public, and providing motivation and coordination to the workers (Mitroff, 2004). To date, the crisis leadership literature has largely focused on the emergence of charismatic or transformational leaders and their effectiveness in crisis (e.g., Bass, 1998; Halverson, et al., 2004). However, at a more basic level, the successful resolution of a crisis can be seen as a series of decisions and judgments (Aguilera, 1990). For example, in a hurricane response effort, data must be gathered regarding the extent of the damage, including the number of people killed or injured and the number of buildings and roadways destroyed. Accordingly, leaders must decide how to distribute personnel, aid supplies, and money. Thus, information assessment and decision making are two core behaviors that are critical for effective crisis leadership (Boin, et al., 2005; Coombs, 2005; Klann, 2003; Leonard, 2004; Useem, Cook, \& Sutton, 2005; Wooten \& James, 2008).

Information assessment includes determining both structural aspects of information flow, such as how to collect and identify data needed for crisis resolution, and procedural aspects of analysis, such as how to prevent errors and reduce biases (Coombs, 2005). Both the type and 
quantity of information gathered are important for the resolution of crisis situations, often requiring the access of multiple, unique data sources (Fearn-Banks, 1996; Hirokawa \& Keyton, 1995). In addition to information gathering and assessment, researchers have argued theoretically (e.g., Boin, et al., 2005) and empirically (Hale, Hale, \& Dulek, 2006; Mintzberg, Raisinghani, \& Theoret, 1976) that decision making is a key task for leaders in crisis situations (Aguilera, 1990; Smart \& Vertinsky, 1977; Wooten \& James, 2008). Crisis decision making involves generating response options, using criteria to evaluate the best course of action, and making recommendations or taking action as a result (Mintzberg, et al., 1976; Sweeny, 2008).

Crisis leaders are required to perform these tasks of information assessment and decision making under tremendous psychological and physical demands (Klann, 2003; Leonard, 2004). The core elements that define a crisis—ambiguity, urgency, and high stakes—also severely constrain the ability of individuals to assess information and make decisions effectively (Boin, et al., 2005; Mumford, Friedrich, Caughron, \& Byrne, 2007; Pearson \& Clair, 1998). As Dutton (1986) notes, it is difficult and perhaps even impossible to achieve a full and complete understanding of the nature, underlying reasons, influence mechanisms, and consequences involved in a crisis. In addition, because leaders in crisis situations are under severe time pressure, they often have less time to acquire, secure, and process information effectively (e.g., Halverson, et al., 2004; Quarantelli, 1988). Furthermore, the life and death stakes involved in the situation are only likely to amplify the sense of time pressure and its desultory effects upon information exploration and decision processing (Mumford, et al., 2007; Sayegh, Anthony, \& Perrewe, 2004; Staw, Sandelands, \& Dutton, 1981). Therefore, the inherent demands of a public health and safety crisis are likely to severely constrain an individual's ability to assess information and make decisions successfully. 
The capability of leaders to assess information and make decisions despite the challenges that crises present is the central topic of the current investigation. Effective performance on these tasks is influenced by many factors, including characteristics of the individual and of the situation (e.g., Pearson \& Clair, 1998; Staw, et al., 1981). Correspondingly, our work explores experiences and capabilities that may influence crisis leader efficacy, including developing and practicing crisis response protocols and being efficacious as a leader in general. We also examine several outcomes to crisis leader efficacy, including the motivation to lead in a crisis, role-taking of leadership positions in a crisis, and the accuracy, difficulty, and confidence associated with making decisions in a crisis context. Finally, we consider certain individual difference characteristics, such as learning goal orientation, intelligence, and divergent thinking, that may influence the relationship between crisis leader efficacy and performance.

\subsection{Contributing factors to crisis leader efficacy}

Procedural forms of crisis preparedness. Currently, great effort and expense are being directed toward the development and testing of crisis response plans in the United States (Lee, Woeste, \& Heath, 2007; Leonard \& Howitt, 2006; Reddick, 2007) and countries around the world. We define procedural preparedness as the outcome of establishing or practicing official emergency response protocols (e.g., developing plans and running drills). Underlying these activities is the assumption that preparedness will enhance the capabilities of individuals to respond successfully to a crisis (Mitroff, 2004; Pearson \& Clair, 1998). However, given the demands of a crisis, such preparation efforts may not inoculate individuals against more pressing "in-the-moment" challenges to assess information and make decisions (Coombs, 2005; Mitroff, 2004). Previous research on the impact of acute stress, for example, indicates that people will revert to their dominant response instead of a recently learned behavior in those circumstances 
(Dickerson \& Kemey, 2004; Staw, et al., 1981). Thus, efforts by leaders to develop and practice crisis response protocols may generally increase their sense of efficacy to perform leadership tasks in a crisis, but these steps may not guarantee successful information assessment and decision making in an actual crisis situation.

Efficacy as a leader in general. Crisis leader efficacy is also likely to be dependent to some degree upon an individual's general leadership capabilities. Leadership efficacy is defined as a person's confident belief that they have the knowledge, skill, and ability to lead others effectively (Anderson, Krajewski, Goffin, \& Jackson, 2008; Hannah, et al., 2008; Paglis \& Green, 2002). Past research indicates that the nature of leadership in general (i.e., leadership in non-crisis situations) is not wholly separable from leadership in crisis situations (Evans, Hammersly, \& Robertson, 2001). For instance, we would expect that many of the basic skills of transformational leadership (e.g., creating vision and inspiring others) would be also important in a crisis context (Evans, et al., 2001). However, compared to ordinary circumstances, the importance of decision making is magnified during crisis and it is more challenging (Mumford, et al., 2007). As Sommer and Pearson (2007) note (citing Parry, 1990), “A crisis affects cognitive, emotional, and behavioral aspects that constrain rationality and cause greater vulnerability than in normal, non-crisis states" (p. 1237). Therefore, general leader efficacy is likely to be a potential contributor to crisis leader efficacy, but it may not be dictate whether an individual is able to competently assess information and make decisions in a crisis fraught with ambiguity, time pressure, and life and death outcomes. Other characteristics, such as the ability to remain calm under duress, may be more relevant to the development of crisis leader efficacy. 1.3 Outcomes of Crisis Leader Efficacy 
Motivation to lead in a crisis. Self-efficacy to perform a particular task or behavior is typically though to lead to higher levels of motivation to do so (Bandura, 1986, 1997). Thus, high self-efficacy to perform the critical crisis leader tasks of information assessment and decision making is likely to be associated with high motivation to demonstrate those skills by leading in an actual crisis. As a construct, motivation to lead is defined as a person's desire to engage in leadership training, roles, responsibilities, and behaviors (Kark \& Van Dijk, 2007; Tschan, Semmer, \& Inversin, 2004). Chan and Drasgow (2001) showed that leader efficacy in general is empirically related to higher motivation to lead. Although motivation to lead specifically in a crisis has not yet been studied, we do know that crisis leaders with high efficacy tend to exert more effort towards actions and persevere when facing difficulties (Bandura, 1997; Gist, 1987; Hannah, et al., 2008). Thus, we would expect that a greater sense of crisis leader selfefficacy would lead to higher motivation to lead in a crisis.

Crisis leader role-taking. Both elements of leadership efficacy and motivation are also likely to impact the degree to which individuals take on leadership roles in a crisis. According to (Bandura, 1986, 1997), self efficacy influences the choices people make and the courses of action they pursue: individuals tend to choose tasks and activities they believe that they can best perform and avoid those they do not feel confident in accomplishing. As noted earlier, the scope of a public health and safety crisis generates opportunities to display leadership in regard to information assessment and decision making throughout the response effort. The degree to which individuals feel efficacious should therefore directly predict the degree to which they take on new or enhanced leadership roles to perform these tasks.

In addition, irrespective of their feelings of efficacy, those individuals who are highly motivated to lead in a crisis can be expected to take on greater levels of leadership roles in a 
crisis. Indeed, previous research has found that motivation to lead positively predicts leadership role-taking in the military (Amit, Lisak, Popper, \& Gal, 2007). Overall, a sense of self-efficacy to assess information and make decisions in crisis situations, as well as a high level of motivation to lead in a crisis more generally, are both likely to contribute to greater leadership role-taking during the occurrence of an actual crisis.

Crisis leader performance. Given that crises include many complex factors that make alternative outcomes difficult to assess or predict, crisis leader performance is often problematic to measure precisely. The decision making literature assumes that leaders who make good decisions are choosing the optimal alternative and are thus most accurate in their choices (Hogarth \& Makridakis, 1981; Mumford \& Connelly, 1991). However, in a crisis, an ideal solution is often not knowable until it is too late, if at all (Batemen, 2008). Although decisionmaking accuracy may be difficult to capture in real-world crisis situations, theoretically, leader efficacy and role-taking should be correlated with more accurate performance (Day, Sin, \& Chen, 2004). In situations in which many of the environmental variables can be controlled, such as in training simulations, it may be possible to see a more transparent and direct relationship between crisis leader efficacy and the accuracy of crisis decisions.

Measures that capture the effectiveness of the decision making process, such as the difficulty and confidence that leaders experience while assessing information and making crisis decisions, are other potentially important indicators of leader performance (e.g., Dearstyne, 2007; Frohman, 2006; Hogarth \& Makridakis, 1981; Weber \& Johnson, 2009). For example, although leading in a crisis is unlikely to be easy for any individual, we expect that some leaders will experience relatively more strain than others when facing the challenges of searching for information, determining and evaluating response choices, and making decisions in this context 
(e.g., Aminilari \& Pakath, 2005; Dearstyne, 2007; Frohman, 2006). This relatively higher level of strain experienced while performing leadership decision-making tasks may be associated with poorer decision quality and lower leadership effectiveness in general (Kayes, 2004; Lipshitz, Klein, Orasanu, \& Salas, 2001; Tett, Guterman, Bleier, \& Murphy, 2000). In addition, the confidence that leaders have in the decisions made under crisis conditions is likely to be based upon their assessment of the quality of the decision-making process involved, including the degree to which alternatives were considered and how well downsides were mitigated (Sweeny, 2008; Tett, et al., 2000). Thus, in addition to the accuracy of decisions made, two additional indicators of crisis decision-making quality are the difficulty and confidence that leaders experience in making their decisions. All of these outcomes should be predicted by the selfefficacy of the individual to perform these tasks.

\subsection{Influences on the efficacy-performance relationship}

There are many characteristics of individuals that may influence the link between their level of crisis leader efficacy and their actual ability to assess information and make decisions in a crisis. In general, a crisis is a unique and extreme situation that may require new ideas or creativity in information assessment and decision-making (Amabile, Hadley, \& Kramer, 2002). Those individuals with high levels of certain types of cognitive characteristics, such as learning goal orientation, intelligence, and divergent thinking, may be more successful at performing these tasks in a crisis, especially if they believe strongly in their efficacy to do so (Brockner \& James, 2008; Dweck, 1990; Gibson, Folley, \& Park, 2009; Sternberg \& Kaufman, 1998; Wood \& Bandura, 1989)

Learning goal orientation. The orientation of a leader toward learning will influence how they process information and make decisions in a crisis. Learning goal orientation is an 
individual trait that reflects being motivated to focus on task mastery for the sake of learning and growing; it is often contrasted to a performance goal orientation that reflects being motivated to demonstrate that one is competent in reference to an external comparison (Dweck, 1990; Hofmann, 1993). With a learning goal orientation, leaders are more open-minded, exploratory and are more adaptive in their response to adverse conditions, which is important for crisis decision making (Brockner \& James, 2008; Cron, Slocum, VandeWalle, \& Fu, 2005; Dweck, 1990). For example, when perceiving a threat, the level of learning goal orientation may contribute to whether the leaders will be open to learning about the situation before making a decision (Brockner \& James, 2008; Button, Mathieu, \& Zajac, 1996). In general, a learning goal orientation is associated with cognitive experimentation or flexibility, which could allow for more successful crisis resolution (Davis, Mero, \& Goodman, 2007).

Intelligence. There are many different definitions and implicit theories of intelligence, including general intelligence, emotional intelligence, and the bioecological model of intelligence (e.g., Barron \& Harrington, 1981; Boyatzis, 2006; Riggio, Murphy, \& Pirozzolo, 2002; Sternberg, 1988). A model of intelligence that is often applied to the leadership domain is the ability to learn and the ability to adapt to environments (Sternberg \& Kaufman, 1998). While intelligence is valued in leadership and decision making in general, intelligence could be especially important in a crisis due to the fast-paced, challenging context (e.g., House \& Aditya, 1997; Judge, Colbert, \& Ilies, 2004).

Divergent thinking. Research suggests that divergent thinking is a key component of creative thought and flexibility in problem solving under various conditions (Gibson, et al., 2009; Guilford, 1959; Mumford, Marks, Connelly, Zaccaro, \& Johnson, 1998). Divergent thinking is signified by the generation of multiple alternative solutions to problems (Gibson, et al., 2009; 
Guilford, 1950; Guilford, 1959; Mumford, et al., 1998). Prior research suggests that the quantity and quality of divergent thinking may be reduced and even disappear under stress, such as extreme time pressure (Abualsamh, Carlin, \& McDaniel, 1990; Amabile, et al., 2002). Nonetheless, the degree to which divergent thinking is incorporated in a crisis is likely to influence how successful the leader is in assessing information and making decisions.

Overall, previous theory and research indicates that a learning goal orientation, intelligence, and divergent thinking ability are all characteristics that should enhance an individual's ability to effectively assess information and make decisions in a crisis. However, these characteristics are likely to be especially important under conditions of high self-efficacy. Self-efficacy should increase the motivation by individuals to harness all of their capabilities to perform effectively in the target situation, and as Wood and Bandura (1989) note, "the richer the repertoire of sub skills that people possess, the easier it is to integrate these skills in the production of new behavior patterns" (p. 363). Therefore, the individual characteristics of learning goal orientation, intelligence, and divergent thinking may serve to enhance the strength of the relationship between crisis leader efficacy and crisis performance.

\subsection{Assessing crisis leader efficacy and performance}

There are many challenges associated with conducting research on crisis phenomena, including gathering data in the moment of catastrophe. Often, data collection cannot be planned in advance because, by their very nature, crises are unanticipated. Once a crisis has begun, researchers are unlikely to gain access to data collection due to the organization's focus on managing the crisis itself or to concerns about its public image or liability (Pearson \& Clair, 1998). It is therefore extremely difficult to gather data across many individuals and situations to broaden the generalizability of findings. 
With such a complex and difficult-to-access phenomenon, determining the appropriate way to measure leaders' information assessment and decision making capacity in a crisis is no easy task. We selected self-efficacy as our measurement construct for three primary reasons. First, self-efficacy has been empirically shown to predict important outcomes, including organizational dynamics (Saks, 1995), training behaviors (Combs \& Luthans, 2007), and work performance (Eden, 1995; Stajkovic \& Luthans, 1998). In particular, a meta-analysis by Stajkovic and Luthans (1998) found a significant correlation between work-related self-efficacy and work performance of .38. This suggests that measuring an individual's self-efficacy to perform in a crisis will in fact be positively correlated with their actual performance in a crisis. Second, a self-efficacy measure locates the construct at the individual level, which allows it to capture variance among different leaders responding to the same crisis. Third, self-efficacy has the advantage of being open to influence (e.g., by training; Dvir, Eden, Avolio, \& Shamir, 2002; Hannah, et al., 2008), rather than a trait-like quality that will remain fixed (Stajkovic \& Luthans, 1998). This makes self-efficacy especially useful not only from the standpoint of identifying and assessing the quality of leadership in crises, but also for improving it.

\subsection{Overview of research objectives}

The three objectives of the current investigation are to develop a scale measure of leader efficacy to assess information and make decisions in a public health and safety crisis, to validate this measure, and to use this measure to explore the nature of crisis leader efficacy. First, we develop and test a measure of crisis leader efficacy, the C-LEAD scale. Scale items are generated based upon the literature and interviews with crisis experts, and refined in a series of preliminary empirical tests. Next, the resulting C-LEAD scale is compared to an existing measure of general leader self-efficacy in two studies using different target populations and decision-making 
settings. In the third study, we elaborate further upon the discriminant and predictive validity of C-LEAD using a simulated crisis decision-making task. From the results of the three studies, we present an initial framework of the nature of crisis leader efficacy in regard to assessing information and making decisions. The framework specifies the likely relationships among crisis preparedness activities, crisis leader efficacy, motivation to lead in a crisis, role-taking of crisis leadership positions, and crisis decision-making performance. We also explicate the potential moderating role that many individual characteristics, including learning goal orientation, intelligence, and divergent thinking, may play in the efficacy-performance relationship.

\section{Development of crisis leader self-efficacy instrument (the C-LEAD scale)}

The items of the C-LEAD scale were originally generated through a review of prior research and interviews with crisis experts. We reviewed the literature on crisis leadership (e.g., Bennis, 2004; Boin, et al., 2005; Klann, 2003) to identify the key elements of effective leader behaviors to include in our scale. In the literature, we found repeated references to the importance of effective information assessment and decision making in a crisis (e.g., Rosenthal \& 't Hart, 1991; Sayegha, Anthony, \& Perrew, 2004). Thus, we decided to address those two critical forms of crisis leader behavior and named our instrument the Crisis Leader Efficacy in Assessing and Deciding scale (C-LEAD).

To develop specific items for the scale, we conducted interviews with women and men who had successfully led others during a previous public health and safety crisis. Interviewees were identified through referrals provided by experts in the field (Biernacki \& Waldorf, 1981). To solicit referrals, we first compiled a list of programs, centers, and institutes that offer training and/or research expertise on the topic of crisis leadership, particularly in the areas of public health and safety in the United States. In total, 28 programs were identified. We next contacted 
the program directors, asking them to recommend individuals who had demonstrated effective leadership during a prior public health or safety crisis. We screened the recommendations for appropriateness for our study, resulting in 57 unique names. Fifty of these leaders (88\%) agreed to be interviewed, 11 women and 39 men. Interviewees came from both federal and state public health departments, emergency response agencies (e.g., fire, police), elected positions, and government agencies. Twenty-two leaders described natural disasters (e.g., hurricanes and floods), 18 described crises intentionally caused by humans (e.g., 9/11 terrorist attacks, anthrax, and riots), and 10 described crises unintentionally caused by humans (e.g., vaccine shortages, disease outbreaks, power outages, and fires).

Semi-structured interviews were conducted by telephone and lasted approximately one hour. In brief, we introduced the project and asked interviewees to describe their roles in resolving the crisis. Next, we probed for information about their actions, emotions, and thoughts during major stages of the crisis response. In the interviews, we also asked about prior crisis experiences and personal characteristics that helped them lead effectively during the crisis. Finally, we asked for general impressions about crisis leadership, differences between crisis and non-crisis leadership, and overall thoughts, impressions, and lessons learned. All interviews were conducted by one of two researchers, and interviewees were assured of confidentiality. Interviews were audio-taped and transcribed. The interview transcripts were coded and analyzed for the critical actions, emotions, and cognitions the interviewees undertook or experienced as they responded to the crisis. From this analysis, we confirmed that successful crisis leaders consistently demonstrated an ability to effectively assess information and make decisions despite the pressures and constraints posed by the crisis situation, and therefore we used this information to generate initial items for the C-LEAD scale. 
In preliminary tests, we varied C-LEAD scale items, instructions, and response sets among 1,688 individuals who were members of a research panel. The protocol used and demographic characteristics of the participants in the pretest studies were very similar to those in Studies 1 and 3, except that the surveys were exclusively used to identify scale items with high internal reliability that still contained external validity to the literature and interviews. Further information on the pretests is available from the first author. At the end of the pretest period, we derived a 9-item C-LEAD scale that showed promising psychometric properties. The original CLEAD scale was used in Studies 1 and 2, and the final scale (after slight modifications to some of the items to enhance their clarity and crisis nature) was used in Study 3. Table 1 contains the C-LEAD scale.

[Insert Table 1 about here]

We conducted three empirical studies to establish the construct validity of the C-LEAD scale. The objective of the first study was to demonstrate that our measure of crisis leader efficacy had strong intrinsic psychometric properties and was distinguishable from an existing measure of general leader efficacy. In this case, we tested C-LEAD and the comparison measures in a general leadership decision-making context to show that C-LEAD could not be used to replace an existing measure of leadership efficacy. In the second study, our objective was to reverse this finding; in this case, we tested the measures in a crisis context to show that C-LEAD was better able to predict decision-making performance in a crisis context than comparison measures. Finally, the objective of the third study was to explore how C-LEAD predicted other outcomes, such as motivational states and leader role-taking, as well as additional aspects of crisis decision-making performance. The third study was also designed to explore potential individual difference moderators of the efficacy-performance relationship. 


\section{Study 1: Initial construct validity of C-LEAD}

The first study was designed to establish the scale's internal reliability, discriminant validity from other measures, and predictive validity of decision-making performance. Since C-LEAD is specific to leadership in a crisis, we sought to differentiate our scale from a measure of leadership in ordinary times. For this comparison, we used a previously validated measure of general leader efficacy. Additionally, we examined the C-LEAD scale in relation to a measure of crisis preparedness (e.g., developing protocols, practicing responses). Finally, we used our scale to predict two important indicators of effective decision-making processes: the difficulty and confidence leaders experience in making decisions. In the current study, a survey was administered to a diverse set of managers in regard to general leadership decisions.

\subsection{Participants and procedures}

Internet-based surveys were distributed through a survey administration company that has a panel of approximately 2.5 million participants across the United States. To ensure that our participants had leadership experience, we required that they were a manager of others at work, as measured by having at least one subordinate reporting to him or her. We did not correspond directly with the participants; the research company administered the survey through their communication channels and provided the data to us without any personally identifying information.

Our sample included 161 men and 121 women (a 23.5\% response rate among those solicited for our survey). The average participant was 45 years old and most (57.8\%) had a fouryear college degree or higher. Participants supervised an average of "between 10 to 14 subordinates" and had been working for their employer for an average of 9.6 years. More than 19 different occupational fields were represented in the sample, including those in the 
private/business $(81.6 \%)$, public/government (8.5\%), non-profit/charitable (4.3\%), and academic (3.5\%) sectors. The majority of participants were in a position of formal authority to direct others at work in the event of a public health and safety emergency (67.7\%).

Participants completed the 9-item C-LEAD scale as well as other scale measures on the survey. The Leadership Self-Efficacy scale (LSE; Paglis \& Green, 2002) assessed the degree to which participants judged that they could accomplish general leadership tasks in the areas of setting a direction for the group, gaining followers' commitment, and overcoming obstacles. The LSE contains 12 items that are averaged together to create a measure of overall leadership efficacy, including, "I can develop plans for change that will take my group in important new directions" and "I can figure out ways for my group to solve any policy or procedural problems hindering our change efforts." Participants also completed a scale created for the study that was designed to assess procedural preparedness for a crisis situation. The scale included six items: (1) "I know who to call if I receive a report of an occurring or impending crisis," (2) "I am unsure what my role and responsibilities would be in a crisis," (3) "I frequently review the crisis response plans that my unit has in place," (4) "I have adequately practiced my unit's crisis response plan," (5) "I keep others at work up-to-date on the best way to reach me in a crisis," and (6) "I do not believe my unit's response plan is the best it can possibly be." Items (2) and (6) were reverse-scored and a mean was calculated. All three measures used 7-point ratings scales that ranged from 1 (Strongly Disagree) to 7 (Strongly Agree).

The survey also included a section devoted to making general leadership decisions. Participants were given a series of four vignettes and asked to make decisions about how the protagonists should proceed (adapted from Kane, Zaccaro, Tremble, \& Masuda, 2002). For example, one of the situations involved assuming the role of a Vice President of a company who 
had to decide how to handle a situation in which an important work group was failing in its mission. Decision options included performing motivational activities with the group and replacing the person in charge of the work group. For each scenario, participants were given four equally viable decision options (Kane, et al., 2002), as well as the option to not make a recommendation. After making a decision, participants indicated the level of difficulty they experienced in making this decision and the level of confidence they felt about it on 5-point Likert response scales that ranged from 1 (Not at All) to 5 (Extremely).

\subsection{Analysis of construct validity properties of C-LEAD scale}

We present the basic psychometric properties of the C-LEAD, LSE, procedural preparedness, decision difficulty and decision confidence measures in Table 2. As shown, the CLEAD measure showed strong internal validity. The correlations among the main study variables also appear in Table 2. As expected, results indicate that C-LEAD was positively correlated with LSE and with procedural preparedness at moderately high levels. As shown, C-LEAD, LSE, and procedural preparedness show some differences in regard to the participants' difficulty and confidence in making general leadership decisions. Specifically, all three variables were significantly negatively correlated with the decision difficulty variable so that higher levels of crisis leader efficacy, general leader efficacy and procedural preparedness were all associated with less difficulty making general leadership decisions. A similar pattern was found for the decision confidence variable. However, as will be presented in the next section, multiple regression analyses showed that only LSE predicted general decision difficulty and confidence when C-LEAD and procedural preparedness were entered as comparison variables.

Separate correlation analyses with the participant demographic variables showed that CLEAD was correlated with whether or not participants were in a position of formal authority in a 
crisis $(r=.19, p<.01)$ and the number of subordinates they had $(r=.20, p<.01)$. C-LEAD was not correlated with any other job or demographic background variables. LSE was correlated with whether or not participants were in a position of formal authority in a crisis $(r=.21, p<.01)$, number of subordinates $(r=.17, p<.01)$, tenure at the employer $(r=.14, p<.05)$, and gender $(r=$ $.20, p<.01$ ), with women having higher general self-efficacy scores relative to men. Procedural preparedness was correlated with whether or not participants were in a position of formal authority in a crisis $(r=.27, p<.01)$, number of subordinates $(r=.20, p<.01)$, and tenure at the employer $(r=.15, p<.05)$.

\section{[Insert Table 2 about here]}

\subsection{Discussion of initial findings regarding construct validity of C-LEAD scale}

The current study provides initial evidence of the construct validity of the C-LEAD scale, as indicated by its psychometric properties and discriminant validity from other related variables (Campbell, 1960). For psychometric properties, the scale showed high internal reliability and a mean and standard deviation consistent with the pre-validated measure of leader self-efficacy. In terms of the discriminant validity of the C-LEAD scale, participant scores on C-LEAD and LSE overlapped by approximately $50 \%$, but this result is consistent with the expectation that crisis leader efficacy is related to, but distinct from, general leader efficacy. Importantly, the study demonstrated differences between C-LEAD and LSE for the prediction of a critical leadership behavior (i.e., making decisions). Results indicated that LSE predicted the level of difficulty and confidence experienced by individuals making decisions in a non-crisis context better than did C-LEAD. Therefore, C-LEAD is not merely a substitute for a measure of general leader selfefficacy as applied to everyday leadership decision-making situations. In general, the findings 
suggest preliminary differences between our measure of crisis leader efficacy and general leader efficacy.

Finally, the C-LEAD scale was significantly and positively correlated with the measure of procedural preparedness at moderate levels, which again is consistent with our expectation. However, in terms of background characteristics, procedural preparedness was solely distinguished from C-LEAD in its significant relationship to tenure at the employer. Procedural preparedness did predict general decision difficulty at a more significant level than C-LEAD, although it was less important to this outcome than LSE. Overall, the results of the current study suggest some distinctions between our measure of crisis leadership efficacy and our measure of procedural preparedness for a crisis, at least in the context of general leadership decisions.

\section{Study 2: Discriminant validity of C-LEAD scale}

Our goal with the second study was to further test the construct validity of the C-LEAD scale, particularly in regard to its discriminant and predictive validity. For the current study, we surveyed crisis responders at a federal agency in the United States who were undergoing an extensive agency-wide simulation of a pandemic influenza outbreak. This study complemented Study 1 by demonstrating the relative usefulness of C-LEAD in predicting decision-making in a crisis context, as compared to measures of general leadership efficacy and procedural forms of crisis preparedness.

\subsection{Participants and procedures}

Survey data were collected at a United States federal agency in association with an ongoing series of crisis preparation exercises. The training exercises involved elaborate simulations in which personnel responded to a pandemic influenza outbreak. A total of 380 representatives from more than fifteen different functional areas and multiple levels of the 
agency were invited to engage in the simulations exercised during data collection. Our sample included 85 participants who completed our primary survey, 51 of whom also completed one measure on a follow-up survey (representing a $22.4 \%$ and $13.4 \%$ response rate of those invited, respectively). The primary sample included 31 men and 52 women (two participants did not indicate gender), with an average age of 45 years of age. The sample was well-educated; $79.4 \%$ had a masters degree or higher. On average, participants supervised at least one subordinate and had been working for their employer for a range of 7 to 8 years. A total of $24.0 \%$ had formal authority to direct others in a public health and safety crisis (note that at the agency, "formal" authority implied a specific title or designation). Participants reported on average that they were "somewhat familiar" with the crisis response protocols of the Department of Homeland Security (DHS) and of their employer. Participants had completed an average of 9.7 preparedness exercises in the past five years and had experienced 2.4 terrorist attacks, major disasters, or other public health and safety emergencies while at work.

To preserve the anonymity of participants' email addresses and identities, a note of introduction including an electronic link to the surveys was distributed by electronic mail to all invitees on our behalf by one of the exercise coordinators. The first survey was distributed over a four-day period prior to the reconvening of the exercise, during which several key issues of the previous exercise phase had been left unresolved. Three days after the new exercise phase ended, using the same delivery method, we distributed a follow-up survey to collect an additional measure. On the first survey, we collected data on C-LEAD, LSE, procedural preparedness, and decision-making variables, as well as participant background information (e.g., participants' crisis experience and training, job characteristics, and demographic traits). The follow-up survey 
included a standard measure of self-presentation bias, the Social Desirability Scale Short Form (SDS; Reynolds, 1982).

Instead of using the vignettes describing general leadership scenarios as a basis for decision-making as in the previous study, in the current study we asked participants to make decisions regarding three issues that were central to both the crisis simulation exercise and to an actual pandemic influenza outbreak. The crisis-related issues were: (a) what stage of alert the U.S. and world should be in, (b) what local communities should do to protect their residents, and (c) how the agency should deploy responders to the field. Pilot testers at the agency confirmed that each of the issues presented to participants were fundamental to an actual pandemic influenza crisis and realistic in the sense that they contained elements of ambiguity (e.g., the exercise data made it unclear if the situation was a true pandemic or not), urgency (e.g., the influenza strain was rapidly spreading across the U.S. in the simulation), and high stakes (e.g., several people had already died from influenza infection in the simulation). We expected that participants would be highly engaged in resolving these issues as each had been designated by the simulation planners as central to the exercises and was unresolved at the time of data collection.

For each of the three crisis issues, participants were asked to choose a recommendation from a set of four options; they were also given the option to not make a recommendation at that time. Previously, experts at the organization had validated that each of the four recommendation options were equally viable and reasonable responses to the issues, and thus participants were told that there were no obvious "right" or "wrong" choices among the options. After each issue was presented and decided upon, participants described how difficult it was to make their 
decision and how confident they were that it was the right one using 5-point Likert scales ranging from 1 (Not at All) to 5 (Extremely).

\subsection{Discriminant validity of C-LEAD scale}

The psychometric properties of the C-LEAD, LSE, procedural preparedness, Social Desirability Scale, decision difficulty, and decision confidence measures are shown in Table 3; C-LEAD again demonstrated strong internal reliability. Table 3 also shows the correlations among the main scale variables collected in Study 2. As shown, C-LEAD was significantly positively correlated with LSE and with procedural preparedness, at levels consistent with Study 1. None of the variables were significantly correlated with the Social Desirability Scale. Furthermore, the results show that C-LEAD was uniquely (negatively) correlated with the level of difficulty experienced in making crisis decisions; LSE and procedural preparedness were not correlated with this variable. C-LEAD was also significantly correlated with greater levels of crisis decision confidence, unlike LSE. Procedural preparedness was correlated with decision confidence, but at a less significant level than C-LEAD.

Separate correlational analyses showed that C-LEAD was positively correlated with the level of familiarity the individuals had with the response protocols of the DHS $(r=.24, p<.05)$ and of their employer $(r=.28, p<.05)$. No significant relationships were found for C-LEAD with the other background characteristics measured. LSE was significantly correlated with the number of subordinates $(r=.30, p<.01)$, whether in a position of formal crisis authority $(r=.23$, $p<.05)$, familiarity with DHS response protocols $(r=.35, p<.01)$, and familiarity with employer response protocols $(r=.38, p<.01)$. Procedural preparedness was significantly correlated with whether in a position of formal crisis authority $(r=.30, p<.01)$, familiarity with DHS response 
protocols $(r=.43, p<.01)$, familiarity with employer response protocols $(r=.51, p<.01)$, and the number of training exercises participated in the past five years $(r=.32, p<.01)$.

[Insert Table 3 about here]

Another view of the differences among C-LEAD, LSE, and procedural preparedness is found by comparing the relationship between these variables and the decision-making variables collected in the first two studies. We had theorized that a measure of general leader self-efficacy (LSE) would be more effective at identifying those who experienced greater difficulty and less confidence while making decisions in a general leadership context than our crisis-specific measure. We further proposed that C-LEAD would be more effective than both LSE and procedural preparedness in identifying those leaders who experienced greater difficulty and less confidence making decisions in a crisis context. As shown in Table 4, we ran two separate regression analyses using the same paradigm of entering C-LEAD, LSE, and procedural preparedness as predictors of the decision difficulty and confidence measures. As shown in Study 1, when the difficulty and confidence measures were collected in the context of general leadership decisions, only LSE remained a significant predictor of these outcomes. However, in Study 2, when the difficulty and confidence measures were collected in the context of crisis leadership decisions, C-LEAD showed superior predictive ability over these comparison measures.

[Insert Table 4 about here]

\subsection{Discussion of initial findings regarding construct validity of C-LEAD scale}

The current study generated several important findings regarding the construct validity of the C-LEAD scale. In regard to psychometric properties, the level of internal reliability found among C-LEAD items was strong within a population comprised of experts in public health and 
safety crises. Moreover, C-LEAD was not correlated with the Social Desirability Scale, which indicates that although it is a self-report measure of efficacy, it does not simply measure the desire to present oneself in a favorable light.

In terms of the discriminant validity of the C-LEAD scale, the findings with participant demographics and background data suggest further differences between our measure of crisis leader efficacy and general leader efficacy. We found that C-LEAD scores continued to be generally independent of the participant background characteristics. On the other hand, LSE was consistently correlated with position-related aspects of leadership, including whether the individual had formal authority to direct others in a crisis and the number of employees supervised. Thus, the findings indicate that efficacy in a crisis context may be less related to positional characteristics of leadership such as the size of one's subordinate pool reporting to individuals, than efficacy in regard to leadership in general.

In addition, the current study provides strong evidence that crisis leadership efficacy is related to, but distinguishable from, more procedural aspects of preparing for a crisis. Although C-LEAD was significantly and positively correlated with procedural crisis preparation, we found important differences between the measures in terms of how they related to background characteristics. For example, the level of procedural preparedness was more strongly related to participants' familiarity with the DHS's and their employer's formal crisis response protocols than was C-LEAD. Furthermore, unlike C-LEAD, higher levels of procedural preparedness were associated with the individual's position of formal authority to lead others in a crisis and the number of preparedness exercises participated in the past five years.

Most notably, the results of Study 2 demonstrate differences between C-LEAD and the comparison measures of LSE and procedural preparedness with regard to the prediction of a 
critical leadership behavior (i.e., making decisions). Previously, Study 1 indicated that LSE predicted the level of difficulty and confidence experienced by individuals making decisions in a non-crisis context better than did C-LEAD. Therefore, measures of general leader self-efficacy are likely to be more useful than C-LEAD in projecting capabilities in everyday leadership decision-making situations. However, in situations involving crisis decisions, as found in Study 2, C-LEAD was more meaningful than a general leader self efficacy measure. In this case, higher C-LEAD scores were significantly correlated with lower levels of difficulty and higher levels of confidence in regard to decisions and recommendations made in a crisis context, but higher LSE scores were not. Finally, the level of the individual's procedural crisis preparedness did not relate to the level of difficulty and confidence they experienced making decisions in a crisis context when compared to C-LEAD. Thus, the results indicate that C-LEAD captures an individuals' ability to assess information and make decisions in a crisis context better than a measure of the extent to which individuals have prepared and practiced response protocols. In total, these results demonstrate that our measure of crisis leader self-efficacy shows promise of being both theoretically and empirically distinct from measures of general leader self-efficacy and procedural forms of crisis preparation.

\section{Study 3: Development of crisis leader framework}

In the third study we sought to extend our empirical and theoretical investigation of the nature of crisis leader efficacy by conducting a longitudinal study with managers that included additional comparison and performance measures. In this study, we investigated the ability of CLEAD to predict several new outcomes: motivation to lead in a crisis, voluntary role-taking of crisis leadership positions, and decision-making accuracy as a leader. We also incorporated individual difference variables as potential moderators of the crisis leader efficacy-performance 
relationship. From these findings, we developed an initial framework of the nature of crisis leader efficacy.

\subsection{Participants and procedures}

The procedures of the current study were very similar to that of Study 1 in that we distributed electronic surveys to organizational leaders through a survey administration company. The main difference is that we collected data on two surveys that were separated by approximately 10 days in time. In total, 300 managers (67.7\% males) with an average age of 42 years completed both surveys (a 37.6\% response rate among those solicited specifically for our study). In general, the participants were well-educated ( $74.7 \%$ had a four-year college degree or higher degree), had worked in their chosen fields for an average of almost 15 years, and typically supervised between 5 and 9 subordinates. Most worked in private organizations (63.7\%), but others worked in public/government (17.0\%), academic (7.3\%), and non-profit (7.0\%) ones. They had experienced an average of at least one public health and safety crisis while in a position of authority at work and had participated in approximately eight crisis training exercises in the past five years. The majority of respondents (64.7\%) reported being in a position to formally direct or supervise other employees in their organization in the event of a public health and safety crisis. Many had some outdoor survival training (49.0\%) and most (64.7\%) had been camping at least five times before, background that is relevant to the crisis decision-making task involved in the study.

To reduce common method bias, we distributed the measures across two separate surveys that were administered approximately 10 days apart. The first survey collected data on crisis leader efficacy (C-LEAD), motivation to lead in a crisis, divergent thinking, procedural preparedness, and participant demographic characteristics. The second survey contained 
measures of learning goal orientation, intelligence, and crisis decision-making performance. The measures of C-LEAD and procedural preparedness were consistent with those used in the previous two studies.

For motivation to lead in a crisis, we adapted the items in Chan and Drasgow's (2001) general measure of motivation to lead. For example, we adapted the original item "I am the type who would actively support a leader but prefers not to be appointed as leader" to "In a crisis, I am the type who would actively support a leader but prefers not to be appointed as leader." In doing so, we reduced the total number of items from 27 in the original scale to 12 in the crisis version for space considerations. The measure used a 7-point rating scale that ranged from 1 (Strongly Disagree) to 7 (Strongly Agree).

For divergent thinking, we used the traditional (Guilford, 1967) paradigm in which participants were asked to generate as many novel uses as possible for common items in a short timeframe. In this case, we provided participants with two minutes each to generate uses for a brick, pencil, and shoe. The divergent thinking responses were coded based on the criteria of fluency (number of valid responses), flexibility (number of categories used), elaboration, and originality (as adjusted by level of fluency). Coding was performed by two researchers. An initial average reliability across categories and uses between the two coders was .78; differences were discussed and resolved. A single performance score was created for each individual by summing across these different characteristics for each of the three uses tested (see Gilhooly, Fioratou, Anthony, \& Wynn, 2007, for more details).

For learning goal orientation, we used a standard measure from (Button, et al., 1996). The measure used a 7-point rating scale that ranged from 1 (Strongly Disagree) to 7 (Strongly Agree). 
For intelligence, we used Sternberg's (1988) checklist of 41 behaviors associated with intelligent people. Participants rated the degree to which the behaviors, such as reasoning logically and well, were characteristic of themselves on a 9-point rating scale that ranged from 1 (Low) to 9 (High).

Leader role-taking and performance were measured using a previously developed wilderness survival task (Pfeiffer \& Jones, 1976). To suit our research purposes, we adapted the scenario and survival decisions involved in the task to include more leadership elements. Specifically, participants were told that while on a team-building retreat in the wilderness of Oregon, they and four of their subordinates, none of whom were experienced campers, had become separated from the group with little equipment (in the original scenario, participants are alone in the wilderness). The simulation involved making challenging decisions in the face of the ambiguity, high stakes, and urgency associated with public health and safety crises (Pearson \& Clair, 1998; Shrivastava, 1993; Sommer \& Pearson, 2007). In the scenario, for example, the situation gets increasingly dire, from simply being lost in the wilderness to facing extreme dehydration, river flooding, and even a bear attack.

After the background of the scenario was presented, participants were presented with a series of 12 decisions to make about how to survive a particular life-threatening circumstance and asked to indicate for which decisions they chose to be the lead decision-maker for the group. For example, the first decision presented was, "Your group has strayed from your party in trackless timber. You have no special signaling equipment. What is the best way to attempt to contact the rest of the campers?" Participants were reminded that in real-world crisis situations, there is often time for only one person to make a decision and were told to assume that another member of the group would make the decision for the group if they choose not to do so. The total 
number of leadership roles taken out of the twelve possible opportunities was summed for each participant.

Following this role-taking task, participants were told to imagine that their subordinates had voted them as lead decision-maker for all decisions and asked them to choose the best option among those offered for each decision. Each of the twelve decisions was repeated, but this time participants were given three answer options, one of which had been determined previously by the original task authors as the correct one. For example, the first decision was presented as, "Your group has strayed from your party in trackless timber. You have no special signaling equipment. The best way to attempt to contact the rest of the campers is to tell your colleagues to: (a) Call for help loudly, but in a low register, (b) Yell or scream as loudly as you can, or (c) Whistle loudly and shrilly." The answer in this case is to call for help loudly but in a low register, as low tones carry farther, especially in dense woodland. The total number of correct decisions was summed for each participant. In addition, the total number of correct decisions as leader was calculated by summing across the twelve decisions for the cases in which the participant had both taken on the leadership role and had answered the question correctly.

After completing the crisis decision-making task, participants were asked a series of questions about their experiences making the 12 decisions. There were four questions that captured the difficulty they experienced making the crisis decisions, such as, "How easy did you find it to make decisions as the lead decision-maker for the group?" Another four questions captured their confidence in the decisions they had made, such as, "How confident are you that your decisions were the correct ones?" The responses were reverse-scored as necessary and standardized so that the means could be captured.

\subsection{Predictive and discriminant validity of C-LEAD scale}


The psychometric properties of the C-LEAD and the other main variables are presented in Table 5. As shown, C-LEAD once again shows strong internal reliability. In addition, as the table indicates, participants typically volunteered to take on the decision-making leadership role for approximately 8 out of 12 decisions. The number of correct decisions for which the individual had taken a leadership role is approximately 3 out of 12 decisions $(24.7 \%)$.

[Insert Table 5 about here]

The correlations among C-LEAD and the main scale variables are also found in Table 5. As shown, C-LEAD was significantly positively correlated with both motivation to lead in a crisis (MTLC) and leader role-taking. As expected, MTLC was also correlated with more frequent role-taking of leadership positions. However, a separate regression analysis indicated that MTLC did not completely mediate the relationship between C-LEAD and leader role-taking, as when both C-LEAD and MTLC were entered, C-LEAD $(\beta=.16, p<.05)$ provided additional predictive power above and beyond the motivation to lead $(\beta=.21, p<.01)$. Thus, C-LEAD was associated with higher motivation to lead in a crisis and both directly and indirectly predicted the degree to which individuals voluntarily took on crisis leadership roles.

In regard to the decision-making variables, higher C-LEAD scores, as collected on the first study, predicted many types of performance variables collected 1-2 weeks later on the wilderness survival task. Consistent with the results of Studies 1 and 2, we found that higher levels of C-LEAD were significantly associated with experiencing less difficulty and greater confidence during the crisis decision-making process. In addition, C-LEAD significantly predicted the number of correct decisions made while in a leadership position. Furthermore, CLEAD $(\beta=.17, p<.01)$ predicted the number of correct in-role decisions even when the degree of outdoor experience $(\beta=.16, p<.05)$ was controlled in a regression analysis. C-LEAD did not 
predict the total number of correct decisions made irrespective of whether the participant had volunteered to be the lead decision-maker for the question.

Finally, Table 5 shows that C-LEAD was positively correlated with procedural preparedness, learning goal orientation, and intelligence at moderate levels. It was not correlated with divergent thinking ability. The measures of procedural preparedness and intelligence showed a similar pattern to C-LEAD in that they predicted the number of leader roles taken, the number of correct decisions made as leader, decision difficulty, and decision confidence. Learning goal orientation significantly predicted leader role-taking, decision difficulty, and decision confidence, but not correct decisions as a leader. Divergent thinking significantly predicted leader role-taking, correct decisions as leader, and decision confidence, but not decision difficulty. None of the variables predicted the total number of correct decisions made irrespective of whether the participant was in the leader role for them.

Table 6 presents the results of a series of regressions in which learning goal orientation, intelligence, and divergent thinking were tested as moderators of the relationships between CLEAD and the main decision performance variables. As shown, all variables showed evidence of moderating the relationships between C-LEAD and the outcomes of decision accuracy, difficulty, and confidence, except in the case of divergent thinking and decision difficulty. Separate analyses were conducted to graph the relationships between C-LEAD and the performance variables at high and low levels of intelligence, learning goal orientation, and divergent thinking, respectively. The results consistently showed a stronger relationship for CLEAD when levels of the individual difference characteristics were high; for example, among people with relatively higher intelligence, there was a steeper regression line between C-LEAD scores and crisis leader accuracy than among people with relatively lower levels of intelligence. 
Similar patterns were shown for the interaction effects of learning goal orientation and divergent thinking upon the relationship between C-LEAD and the performance variables.

\section{[Insert Table 6 about here]}

\subsection{Discussion of findings regarding crisis leader efficacy framework}

The results of the current study reinforce the previous studies' findings and provide additional evidence for the construct validity of the C-LEAD scale in terms of its internal reliability, predictive validity, and discriminant validity. As consistent with the findings of Studies 1 and 2, we found that procedural crisis preparedness was significantly correlated with C-LEAD scores. Thus, efforts to develop and practice crisis response protocols are likely to result in higher levels of self- efficacy to assess information and make decisions in a crisis context, as one might hope and expect.

In addition, C-LEAD predicted an important motivational state, the desire to take on leadership positions in a crisis situation. This finding is in line with the expectation that individuals who feel efficacious to perform a certain behavior in a certain context would be willing and eager to demonstrate these capabilities (e.g., Hannah, et al., 2008; Paglis \& Green, 2002). However, high levels of motivation do not always translate into behavioral confirmation (e.g., Gist, 1987). In this case, we did find that motivation to lead in a crisis predicted greater levels of leader role-taking, even though the measures were separated in time and space. It could be that crisis situations, which are urgent and important, create a greater impetus for people to act upon their underlying motivational states than ordinary situations. We also found that C-LEAD directly predicted leader role-taking irrespective of the level of motivation to lead in a crisis. Thus, merely holding a strong belief in their skill to assess information and make decisions in a crisis influenced the degree to which people volunteered for decision-making leadership roles. 
With regard to the performance variables, we replicated the previous findings that $\mathrm{C}$ LEAD predicted the difficulty and confidence leaders experience in the crisis decision-making process. In addition, while C-LEAD did not predict the total number of correct decisions, it did predict the number of correct decisions made while a leader. Thus, C-LEAD predicts accuracy when it is most likely to count—when the decision-maker is in the position to enact his or her decisions as leader.

We also learned more about the nature of crisis leader efficacy and its relationship to performance through the inclusion of potential individual difference moderators. In general, CLEAD was positively correlated with learning goal orientation and intelligence, but not divergent thinking. Overall, regression analyses showed that higher levels of each of these characteristics may serve to enhance the relationship between crisis leader efficacy and crisis decision-making performance. As Woods and Bandura (1989) note, the most effective way that individuals develop a sense of self-efficacy is through mastery experiences. It is possible that those individuals who have a strong learning goal orientation, are highly intelligent, or can think divergently have a more extensive set of mastery experiences to draw upon as they think about their crisis leader efficacy. Thus, they are not only more likely to perform well on decisionmaking tasks given their natural cognitive capabilities and inclinations, they are also likely to be more accurate in their self-assessment on the C-LEAD scale.

From the results of the three studies, we generated an initial framework of the nature of crisis leader efficacy in regard to assessing information and making decisions. As shown in Figure 1, the framework specifies the likely relationships among crisis preparedness activities, crisis leader efficacy, motivation to lead in a crisis, role-taking of crisis leadership positions, and crisis decision-making performance. Finally, it indicates the moderator role that the individual 
characteristics of learning goal orientation, intelligence, and divergent thinking may play between efficacy and performance.

\section{General discussion}

\subsection{Summary of main findings}

Overall, the results of the three studies provide strong evidence that we achieved our research objectives. The C-LEAD scale was developed through a literature review and interviews with expert individuals who had successfully led others during past public health and safety crises. Based on this background work, we focused our measure of crisis leader efficacy on the critical behaviors of information assessment and decision-making under the conditions of ambiguity, high stakes, and urgency present in a crisis (Pearson \& Clair, 1998). Extensive pretests were conducted to ensure the scale demonstrated strong face validity and internal reliability. To our knowledge, no other scale to evaluate leadership in a crisis has been developed in a similarly rigorous empirical manner.

Next, we established the construct validity of the C-LEAD scale through a series of three studies that incorporated a variety of comparison measures. In the first two studies, we compared our measure of crisis leader efficacy to one of general leader efficacy. We found that the two measures, although correlated as expected, nonetheless predicted decision-making difficulty and confidence differentially. Specifically, our measure of crisis leader efficacy was better at predicting difficulty and confidence in making crisis decisions than a measure of general leader efficacy. The reverse was found when general leadership decisions were considered; the existing measure outshone C-LEAD in predicting performance in a general decision context. Thus, our measure of crisis leader efficacy contributes to the field a new tool for assessing and predicting leadership in a crisis context, but does not remove the need for separate measures of leadership 
capabilities in ordinary times. Our tool may capture aspects of leadership that are especially critical in a crisis and that may be missed with other existing measures of leadership efficacy (e.g., Anderson, et al., 2008; Hannah, et al., 2008; Paglis \& Green, 2002; Tett, et al., 2000; Yukl, 1999).

As part of the construct validation process, we also captured crisis preparedness in terms of procedural aspects of crisis response (Coombs, 2005; Mitroff, 2004). These more formal activities to establish and rehearse appropriate behaviors in a crisis (such as establishing protocols and practicing them through exercises) should correlate with greater levels of crisis leader efficacy, and indeed they do. However, procedural crisis preparedness does not overlap completely with our measure of crisis leader efficacy. This implies that simply knowing and practicing crisis response plans may be inadequate to ensure that leaders are ready to effectively assess information and make decisions in a crisis. Furthermore, we found in the third study that our measure of C-LEAD predicted the number of correct decisions made as a leader more powerfully than the measure of procedural preparedness, although both were correlated with this outcome. Being able to predict decision making more accurately in crisis can make the difference between life and death.

The third study also allowed us to elaborate upon the nature of crisis leader efficacy and its relationship to motivational states, leader role-taking behaviors, individual characteristics, and multiple types of crisis decision-making performance. In the study, we showed that C-LEAD predicted both motivation to lead in a crisis and the actual volunteering for crisis leader roles. Furthermore, we extended the findings of previous studies regarding the predictive power of CLEAD on decision-making difficulty and confidence to show it also predicted decision accuracy as a leader. Thus, our measure of crisis leader efficacy was useful in estimating how motivated 
individuals were to lead others in a crisis, how often they took on that role, and how well they did once they were in it. Furthermore, our exploration of traits such as learning goal orientation and intelligence showed the potential moderating role of these characteristics on the efficacyperformance relationship. From these findings, we have established an initial framework of the nature of crisis leader efficacy that may inspire and guide future research on the topic.

\subsection{Study limitations and future research}

One potential limitation of the current research stems from the self-report method used for C-LEAD and other measures in the study. Any measure of self-efficacy is by nature selfreported, but this does raise the possibility of self-presentation bias. The lack of relationship between C-LEAD and the Social Desirability Scale in Study 2 decreases the potency of this concern, but remains a potential limitation that should be addressed in future research. The use of expert ratings to assess the accuracy of performance in Study 3 also provides some evidence that the use of externally-evaluated performance measures will be predicted by C-LEAD. However, it would be useful to measure additional aspects of individuals and their performance through methods such as archival data and peer or supervisor ratings to further mitigate potential issues related to self-reported measures. In addition, all measures were collected on the same survey (except for SDS) in the first two studies, which creates the possibility of common method bias. In Studies 1 and 2, we tried to mitigate this concern as much as possible by separating our comparison measures of C-LEAD, LSE, and procedural preparedness from each other and from the decision-making variables on the surveys. However, it is only in Study 3 that we achieved a more robust separation of measures through the issuance of two surveys 10 days apart. Nonetheless, it would be ideal to collect the key measures across additional time periods to isolate them further. 


\subsection{Synthesis and implications for research and practice}

For decades, academics have been limited in their empirical research on crisis leadership due to both practical and theoretical limitations (e.g., Pearson \& Clair, 1998; Sweeny, 2008). With C-LEAD, we now have a tool to specifically evaluate crisis leaders where one has not existed before. Theory building and testing can now be pushed to a new frontier, where the unique characteristics of leadership in a crisis can be assessed rather than applying metrics applicable to general situations. The ability of C-LEAD to predict outcomes better than a general measure of leadership efficacy or procedural preparedness underscores the importance of a crisis-specific leadership tool.

Furthermore, this tool makes a valuable contribution to the practice of leader training and development, as it can be used to identify the capabilities of leaders in advance of a crisis so that interventions to improve efficacy can be made (Combs \& Luthans, 2007; Pearson \& Mitroff, 1993). In addition, since C-LEAD predicts leader role taking, crisis training programs can be structured more efficiently to target those most likely to volunteer to take on crisis leader roles.

Overall, the current research underscores our need to develop a better understanding of what "good" crisis leaders look like and how to identify, encourage, and improve the capabilities of all those who might respond to a public health and safety crisis. Future research using CLEAD will continue to refine our understanding of the nature and measurement of crisis leader efficacy and ultimately may enhance our nation's and the world's ability to prevent and respond to these catastrophic events. 


\section{References}

Abualsamh, R. A., Carlin, B., \& McDaniel, R. R. (1990). Problem structuring heuristics in strategic decision making. Organizational Behavior and Human Decision Processes, 45(2), 159-174.

Aguilera, D. C. (1990). Crisis intervention: Theory and methodology (6th ed.). St. Louis, MO: C. V. Mosby.

Amabile, T. M., Hadley, C. N., \& Kramer, S. J. (2002). Creativity under the gun. Harvard Business Review, 80(8), 52-61.

Aminilari, M., \& Pakath, R. (2005). Searching for information in a time-pressured setting: experiences with a Text-based and an Image-based decision support system. Decision Support Systems, 41(1), 37-68.

Amit, K., Lisak, A., Popper, M., \& Gal, R. (2007). Motivation to lead: Research on the motives for undertaking leadership roles in the Israel Defense Forces (IDF). Military Psychology, 19(3), 137-160.

Anderson, D. W., Krajewski, H. T., Goffin, R. D., \& Jackson, D. N. (2008). A leadership selfefficacy taxonomy and its relation to effective leadership. The Leadership Quarterly, 19(5), 595-608.

Bandura, A. (1982). Self-efficacy mechanism in human agency. American Psychologist, 37(2), 122-147.

Bandura, A. (1986). Social foundations of thought and action: A social cognitive theory. . Englewood Cliffs, NJ: Prentice Hall.

Bandura, A. (1997). Self-efficacy: The exercise of control. New York: Freeman.

Barron, F., \& Harrington, D. M. (1981). Creativity, intelligence, and personality. Annual Review of Psychology, 32, 439-476. 
Bass, B. M. (1998). Transformational leadership: Industrial, military, and educational impact. Mahwah, N.J.: Lawrence Erlbaum Associates.

Bateman, T. O. M. (2008). Leading for results: Brief-but-powerful lessons from Katrina and Iraq. Organizational Dynamics, 37(4), 301-312.

Bennis, W. (2004). The crucibles of authentic leadership In J. Antonakis, A. T. Cianciolo \& R. J. Sternberg (Eds.), The Nature of Leadership. Thousand Oaks, CA: Sage.

Biernacki, P., \& Waldorf, D. (1981). Snowball sampling: Problems and techniques of chain referral sampling. Sociological Methods \& Research, 10, 141-163.

Billings, R. S., Milburn, T. W., \& Schaalman, M. L. (1980). A model of crisis perception: A theoretical and empirical analysis. Administration Science Quarterly, 25(2), 300-316.

Boin, A., 't Hart, P., Stern, E., \& Sundelius, B. (2005). The politics of crisis management: Understanding public leadership when it matters most. Cambridge: Cambridge University Press.

Boyatzis, R. E. (2006). Using tipping points of emotional and cognitive intelligence to predict financial performance of leaders. Psicothema, 8, 124-131.

Brockner, J., \& James, E. H. (2008). Toward an understanding of when executives see crisis as opportunity. Journal of Applied Behavioral Science, 44(1), 94-115.

Button, S. B., Mathieu, J. E., \& Zajac, D. M. (1996). Goal orientation in organizational research: A conceptual and empirical foundation. Organizational Behavior and Human Decision Processes, 67(1), 26-48.

Campbell, D. T. (1960). Recommendation for APA test standards regarding constructs, trait, or discriminant validity. American Psychologist, 15, 546-553. 
Chan, K. Y., \& Drasgow, F. (2001). Toward a theory of individual differences and leadership: Understanding the motivation to lead. Journal of Applied Psychology, 86(3), 481-498.

Combs, G. M., \& Luthans, F. (2007). Diversity training: Analysis of the impact of self-efficacy. Human Resources Quarterly, 18, 91-120.

Coombs, W. T. (2005). Ongoing crisis communication: Planning, managing, and responding. Thousand Oaks, CA: Sage

Cron, W., Slocum, J., VandeWalle, D., \& Fu, F. (2005). The role of goal orientation, negative emotions and goal setting when initial performance falls short of one's performance goal. Human Performance, 18, 55-81.

Davis, W. D., Mero, N., \& Goodman, J. M. (2007). The interactive effects of goal orientation and accountability on task performance. Human Performance, 20(1), 1-21.

Day, D. V., Sin, H. P., \& Chen, T. T. (2004). Assessing the burdens of leadership: Effects of formal leadership roles on individual performance over time. Personal Psychology, 57, $573-605$.

Dearstyne, B. (2007). The FDNY on 9/11: Information and decision making in crisis. Government Information Quarterly, 24, 29-46.

Dickerson, S. S., \& Kemey, M. E. (2004). Acute stressors and cortisol responses: A theoretical integration and synthesis of laboratory research. Psychological Bulletin, 130, 355-391.

Dutton, J. E. (1986). The processing of crisis and non-crisis strategic issues. The Journal of Management Studies, 23(5), 501-517.

Dvir, T., Eden, D., Avolio, B. J., \& Shamir, B. (2002). Impact of transformational leadership on follower development and performance: a field experiment. Academy of Management Journal, 45(4), 735-744. 
Dweck, C. S. (1990). Self-theories and goals: Their role in motivation, personality and development. In A. R. Dienstbier (Ed.), Nebraska symposium on motivation: Perspectives on motivation (Vol. 38, pp. 199-235). Lincoln: University of Nebraska Press.

Eden, D. Z., Y. (1995). Seasickness as a self-fulfilling prophecy: Raising self-efficacy to boost performance at sea. Journal of Applied Psychology, 80, 628-635.

Evans, C., Hammersly, G. O., \& Robertson, M. (2001). Assessing the role and efficacy of communication strategies in times of crisis. Journal of European Industrial Training, 25, 297-309.

Fearn-Banks, K. (1996). Crisis communications: A casebook approach. Mahwah, NJ: Lawrence Elrlbaum.

Fowler, K. L., Kling, N. D., \& Larson, M. D. (2007). Organizational preparedness for coping with a major crisis or disaster. Business \& Society, 46, 88-103.

Frohman, D. (2006). Leadership under fire. Harvard Business Review, 84, 124-131.

Gibson, C., Folley, B. S., \& Park, S. (2009). Enhanced divergent thinking and creativity in musicians: A behavioral and near-infrared spectroscopy study. Brain \& Cognition, 69(1), 162-169.

Gilhooly, K. J., Fioratou, E., Anthony, S. H., \& Wynn, V. (2007). Divergent thinking: strategies and executive involvement in generating novel uses for familiar objects. British Journal of Psychology, 98(Part 4), 611-625.

Gist, M. E. (1987). Self-efficacy: Implications for organizational behavior and human resource management. Academy of Management Review, 12(3), 472-485. 
Gorge, M. (2006). Crisis management best practice: Where do we start from? Computer Fraud \& Security, 2006(6), 10-13.

Guilford, J. P. (1950). Creativity. American Psychologist, 5, 444-454.

Guilford, J. P. (1959). Traits of creativity. In H. H. Anderson \& M. S. Anderson (Eds.), Creativity and its cultivation, addresses presented at the interdisciplinary symposia on creativity (pp. 142-161). New York: Harper.

Guilford, J. P. (1967). The nature of human intelligence. New York: McGraw-Hill.

Hale, J. E., Hale, D. P., \& Dulek, R. E. (2006). Decision processes during crisis responses: An exploratory investigation. Journal of Managerial Issues, VXIII, 301-320.

Halverson, S. K., Murphy, S. E., \& Riggio, R. E. (2004). Charismatic leadership in crisis situations: A laboratory investigation of stress and crisis. Small Group Research, 35(5), 495-514.

Hannah, S. T., Avolio, B. J., Luthans, F., \& Harms, P. D. (2008). Leadership efficacy: Review and future directions. Leadership Quarterly, 19, 669-692.

Hirokawa, R. Y., \& Keyton, J. (1995). Perceived facilitators and inhibitors of effectiveness in organizational work teams. Management Communication Quarterly, 8(Measuring the Efficacy 23), 424-446.

Hofmann, D. A. (1993). The influence of goal orientation on task performance: A substantively meaningful suppressor variable. Journal of Applied Social Psychology, 23(22), 18271846.

Hogarth, R. M., \& Makridakis, S. (1981). Forecasting and planning: An evaluation. Management Science, 27, 115-138. 
House, R. J., \& Aditya, R. N. (1997). The social scientific study of leadership: Quo vadis? Journal of Management, 23(3), 409-473.

Hunt, J. G., Boal, K. B., \& Dodge, G. E. (1999). The effects of visionary and crisis-responsive charisma on followers: An experimental examination of two kinds of charismatic leadership. Leadership Quarterly, 10(3), 423-448.

Judge, T. A., Colbert, A. E., \& Ilies, R. (2004). Intelligence and leadership: A quantitative review and test of theoretical Propositions. Journal of Applied Psychology, 89(3), 542552.

Kane, T. D., Zaccaro, S. J., Tremble, T. R., Jr., \& Masuda, A. D. (2002). An examination of the leader's regulation of groups. Small Group Research, 33, 65-120.

Kark, R., \& Van Dijk, D. (2007). Motivation to lead, motivation to follow: The role of the selfregulatory focus in leadership processes. Academy of Management Review, 32(2), 500528.

Kayes, D. C. (2004). The 1996 Mount Everest climbing disaster: The breakdown of learning in teams. Human Relations, 57(10), 1263-1284.

Klann, G. (2003). Crisis leadership. Greensboro, NC: Center for Creative Leadership.

Lee, J., Woeste, J., \& Heath, R. L. (2007). Getting ready for crises: Strategic excellence. Public Relations Review, 33, 334-336.

Leonard, H. B. D. (2004). Leadership in crisis situations The encyclopedia of leadership (In J. Burns, G. R. Goethals, \& G. J. Sorenson, (2004) ed.). Great Barrinton, Ma: Berkshire Publishing Group. 
Leonard, H. B. D., \& Howitt, A. (2006). Katrina as prelude: Preparing for and responding to Katrina-Class disturbances in the United States: Testimony to U.S. Senate Committee, . Journal of Homeland Security and Emergency Management, 3, 1-20.

Lipshitz, R., Klein, G., Orasanu, J., \& Salas, E. (2001). Focus article: Taking stock of naturalistic decision making. Journal of Behavioral Decision Making, 14, 331-352.

Mintzberg, H., Raisinghani, D., \& Theoret, A. (1976). The structure of unstructured decision processes. Administration Science Quarterly, 21, 246-275.

Mitroff, I. I. (2004). Crisis leadership: Planning for the unthinkable. Hoboken, NJ: Wiley.

Mitroff, I. I., \& Alpaslan, M. C. (2003). Preparing for evil. Harvard Business Review, 81(4), $109-115$.

Mumford, M. D., \& Connelly, S. (1991). Leaders as creators: Leader performance and problem solving in ill-defined domains. The Leadership Quarterly, 2, 289-315.

Mumford, M. D., Friedrich, T. L., Caughron, J. J., \& Byrne, C. L. (2007). Leader cognition in real-world settings: How do leaders think about crises? Leadership Quarterly, 18(6), 515543.

Mumford, M. D., Marks, M. A., Connelly, M. S., Zaccaro, S. J., \& Johnson, J. F. (1998). Domain-based scoring in divergent-thinking tests: Validation evidence in an occupational sample. Creativity Research Journal, 11(2), 151-163.

Paglis, L. L., \& Green, S. G. (2002). Leadership self-efficacy and managers' motivation for leading change. Journal of Organizational Behavior, 23, 215-235.

Pang, A., Cropp, F., \& Cameron, G. T. (2006). Corporate crisis planning: Tensions, issues, and contradictions. Journal of Communication Management, 10, 371-389.

Parry, G. (1990). Coping with crises. New York: Routledge, Chapman, and Hall. 
Pearson, C. M., \& Clair, J. A. (1998). Reframing crisis management. Academy of Management Review, 23(1), 59-76.

Pearson, C. M., \& Mitroff, I. I. (1993). From crisis prone to crisis prepared: A framework for crisis management. The Executive, 7(1), 48-59.

Pfeiffer, J. W., \& Jones, J. E. (Eds.). (1976). The 1976 annual handbook for group facilitators. La Jolla, CA: University Associates.

Pillai, R., \& Meindl, J. R. (1998). Context and charisma: A "meso" level examination of the relationship of organic structure, collectivism, and crisis to charismatic leadership. Journal of Management, 24(5), 643-671.

Quarantelli, E. L. (1988). Disaster crisis management: A summary of research findings. Journal of Management Studies, 25, 373-385.

Reddick, C. G. (2007). Homeland security preparedness and planning in US city governments: A survey of city managers. Journal of Contingencies \& Crisis Management, 15, 157-167.

Reynolds, W. M. (1982). Development of reliable and valid short forms of the Marlowe-Crowne Social Desirability Scale. . Journal of Clinical Psychology, 38, 119-125.

Riggio, R. E., Murphy, S. E., \& Pirozzolo, F. J. (2002). Multiple intelligences and leadership. Mahwah, NJ: Lawrence Erlbaum Associates.

Rosenthal, U., \& 't Hart, P. (1991). Experts and decision makers in crisis situations. Knowledge: Creation, Diffusion, Utilization, 12(4), 350.

Saks, A. M. (1995). Longitudinal field investigation of the moderating and mediating effects of self-efficacy on the relationship between training and newcomer adjustment. Journal of Applied Psychology, 80, 211-225. 
Sayegh, L., Anthony, W. P., \& Perrewe, P. L. (2004). Managerial decision-making under crisis: The role of emotion in an intuitive decision process. Human Resource Management Review, 14(2), 179-199.

Sayegha, L., Anthony, W. P., \& Perrew, P. L. (2004). Managerial decision-making under crisis: The role of emotion in an intuitive decision process. Human Resource Management Review, 14(2), 179-200.

Schoenberg, A. (2005). Do crisis plans matter? A new perspective on leading during a crisis. Public Relations Quarterly, 50, 2-7.

Shrivastava, P. (1993). Crisis theory/practice: Towards a sustainable future. Industrial and Environmental Crisis Quarterly, 7, 23-42.

Smart, C., \& Vertinsky, I. (1977). Designs for crisis decision units. Administrative Science Quarterly, 22(4), 640-657.

Sommer, A., \& Pearson, C. M. (2007). Antecedents of creative decision making in organizational crisis: A team-based simulation. Technological Forecasting and Social Change: An International Journal, 74, 1234-1251.

Stajkovic, A. D., \& Luthans, F. (1998). Self-efficacy and work related performance: A metaanalysis. Psychological Bulletin, 124, 240-261.

Staw, B. M., Sandelands, L. E., \& Dutton, J. E. (1981). Threat rigidity effects in organizational behavior: A multilevel analysis. Administrative Science Quarterly, 26(4), 501-524.

Sternberg, R. J. (1988). The triarchic mind: A new theory of human intelligence. New York: Viking.

Sternberg, R. J., \& Kaufman, J. C. (1998). Human abilities. Annual Review of Psychology, 49(1), 479-502. 
Sweeny, K. (2008). Crisis decision theory: Decisions in the face of negative events. Psychological Bulletin, 134(1), 61-76.

Tett, R. P., Guterman, H. A., Bleier, A., \& Murphy, P. J. (2000). Development and content validation of a 'hyperdimensional' taxonomy of managerial competence. Human Performance, 13(3), 205-251.

Tichy, N. M., \& Bennis, W. G. (2007). Making judgment calls. Harvard Business Review, 85(10), 94-102.

Tschan, F., Semmer, N. K., \& Inversin, L. (2004). Work related and "private" social interactions at work. [Empirical]. Social Indicators Research, 67, 145-182.

Useem, M., Cook, J., \& Sutton, L. (2005). Developing leaders for decision making under stress: Wildland firefighters in the South Canyon Fire and its aftermath. Academy of Management Learning \& Education, 4, 461-485.

Vaughan, D. (1996). The Challenger launch decision: Risky technology, culture, and deviance at NASA. Chicago: University of Chicago Press.

Weber, E. U., \& Johnson, E. J. (2009). Mindful judgment and decision making. Annual Review of Psychology, 60(1), 53-85.

Wood, R. E., \& Bandura, A. (1989). Social cognitive theory of organizational management. Academy of Management Review, 14, 361-384.

Wooten, L. P., \& James, E. H. (2008). Linking crisis management and leadership competencies: The role of human resource development. Advances in Developing Human Resources, 10(3), 352-379.

Yukl, G. (1999). An evaluation of conceptual weaknesses in transformational and charismatic leadership theories. Leadership Quarterly, 10, 285-305. 
Figure 1

A theoretical model of the relationships among procedural crisis training and preparedness, crisis leader self-efficacy, motivation to lead in a crisis, crisis leadership role-taking, individual differences, and crisis decision-making performance.

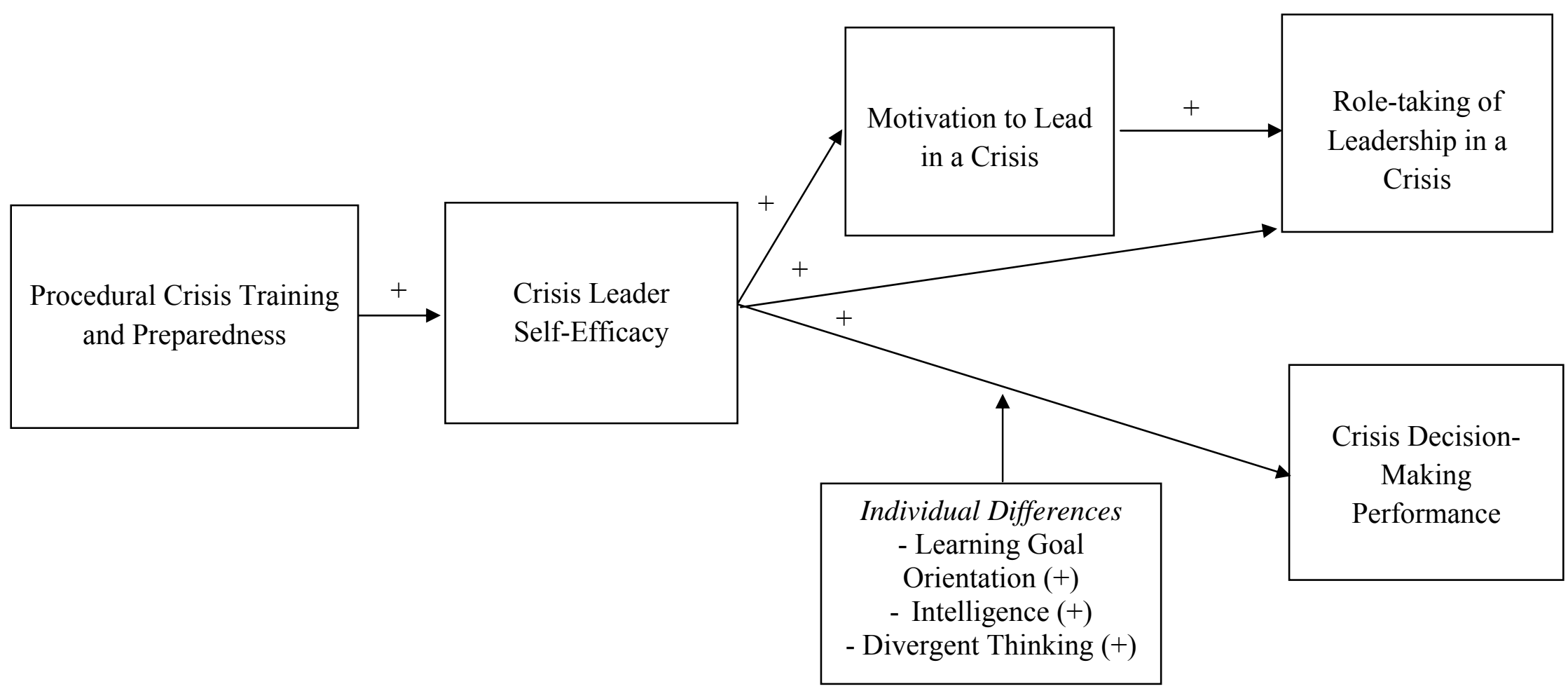




\section{Table 1}

The Crisis Leader Efficacy in Assessing and Deciding (C-LEAD) Scale

1. I can anticipate the political and interpersonal ramifications of my decisions and actions.

2. I can summarize the key issues involved in a situation to others regardless of how much data I have.

3. I can make decisions and recommendations even when I don't have as much information as I would like.

4. I can assess how the members of the general public are being impacted by my unit's actions or inactions during times of adversity.

5. I can determine which information is critical to relay to other units in advance of them requesting it.

6. I can keep others abreast of my work activities without over-informing or under-informing them.

7. I can make decisions and recommendations even under extreme time pressure.

8. I can estimate the potential deaths and injuries that may occur as the result of my decisions or recommendations at work.

9. I can modify my regular work activities instantly to respond to an urgent need. 
Table 2

Study 1: Correlations between C-LEAD and other variables and their psychometric properties

\begin{tabular}{|c|c|c|c|c|c|c|c|}
\hline & 1 & 2 & 3 & 4 & 5 & Mean & $S D$ \\
\hline 1. C-LEAD & .83 & & & & & 4.99 & .94 \\
\hline 2. LSE & $.54 * *$ & .95 & & & & 5.77 & .82 \\
\hline 3. Procedural preparedness & $.43 * *$ & $.61 * *$ & .76 & & & 5.18 & .96 \\
\hline General decision difficulty & $-.13^{*}$ & $-.27 * *$ & $-.16^{* *}$ & .51 & & 1.83 & .55 \\
\hline 5. General decision confidence & $.29 * *$ & $.41 * *$ & $.28 * *$ & $-.53 * *$ & 70 & 3.81 & .67 \\
\hline
\end{tabular}

Note. $N=282$ for all correlations. LSE = Leadership Self-Efficacy scale. Reliability coefficients (alphas) are presented along the diagonal.

$* p<.05 . * * p<.01$. 
Table 3

Study 2: Correlations between C-LEAD and other variables and their psychometric properties

\begin{tabular}{|c|c|c|c|c|c|c|c|c|}
\hline & 1 & 2 & 3 & 4 & 5 & 6 & Mean & $S D$ \\
\hline 1. C-LEAD & .81 & & & & & & 5.36 & .73 \\
\hline 2. LSE & $.49 * *$ & .93 & & & & & 5.61 & .76 \\
\hline 3. Procedural preparedness & $.32 * *$ & $.37 * *$ & .81 & & & & 4.78 & 1.05 \\
\hline 4. Social Desirability Scale & .08 & .27 & .10 & .73 & & & 8.71 & 2.68 \\
\hline 5. Crisis decision difficulty & $-.27 *$ & -.10 & -.16 & .11 & .64 & & 2.06 & .77 \\
\hline 6. Crisis decision confidence & $.34 * *$ & .12 & $.23 *$ & -.06 & $-.55 * *$ & .82 & 2.99 & .92 \\
\hline
\end{tabular}

Note. $N=73-83$ for all correlations except those involving the Social Desirability Scale $(N=50-51)$. Reliability coefficients (alphas) are presented along the diagonal.

$* p<.05 . * *<.01$ 
Table 4

Studies 1 and 2: Comparative view of regression analyses using decision difficulty and confidence variables

Study 1:

General Decision Context

Decision Difficulty

C-LEAD

LSE

Procedural preparedness

Decision Confidence

C-LEAD

LSE

Procedural preparedness
.02

$-.28 * *$

.00

.09

$.33 * *$

.04
Study 2:

Crisis Decision Context

$-.23+$

.03

$-.07$

$.31^{*}$

$-.08$

.15

Note. Enter entry method used. $N=282$ for Study 1 and $N=79$ for Study 2 .

$+\mathrm{p}<.10 ; * p<.05 ; * * p<.01$ 
Table 5

Study 3: Correlations between C-LEAD and other variables and their psychometric properties

\begin{tabular}{|c|c|c|c|c|c|c|c|c|c|c|c|c|c|}
\hline & 1 & 2 & 3 & 4 & 5 & 6 & 8 & 9 & 10 & 11 & 12 & Mean & $S D$ \\
\hline 1. C-LEAD & .88 & & & & & & & & & & & 5.46 & .90 \\
\hline 2. Procedural preparedness & $.42 * *$ & .84 & & & & & & & & & & 4.54 & .87 \\
\hline 3. Motivation to lead in a crisis & $.52 * *$ & $.44 * *$ & .84 & & & & & & & & & 5.11 & .85 \\
\hline 4. Learning goal orientation & $.38 * *$ & $.41 * *$ & $.15^{*}$ & .92 & & & & & & & & 5.84 & .81 \\
\hline 5. Intelligence & $.52 * *$ & $.41 * *$ & $.15^{* *}$ & $.63 * *$ & .97 & & & & & & & 7.26 & .98 \\
\hline 6. Divergent thinking ability & .09 & $.20 * *$ & .10 & $.36 * *$ & $.39 * *$ & .90 & & & & & & 32.57 & 17.78 \\
\hline 7. Number of crisis leader roles taken & $.26 * *$ & $.29 * *$ & $.23^{* *}$ & $.22 * *$ & $.17^{* *}$ & $.29 * *$ & -- & & & & & 8.41 & 3.18 \\
\hline 8. Total correct decisions & .01 & .05 & .11 & -.02 & -.00 & .08 & $.12 *$ & -- & & & & 4.27 & 1.73 \\
\hline 9. Correct decisions as crisis leader & $.20 * *$ & $.22 * *$ & $.18^{* *}$ & $.12 *$ & .10 & $.24 * *$ & $.65^{* *}$ & $.74 * *$ & -- & & & 2.96 & 2.05 \\
\hline 10. Crisis decision difficulty & $-.21 * *$ & $-.24 * *$ & -.10 & $-.18 * *$ & $-.14 *$ & $-.17 * *$ & $-.27 * *$ & $-.17 * *$ & $-.27 * *$ & .60 & & .00 & .73 \\
\hline 11. Crisis decision confidence & $.27 * *$ & $.26^{* *}$ & $.17 * *$ & $.25 * *$ & $.21 * *$ & $.33 * *$ & $.48 * *$ & $.15^{* *}$ & $.39 * *$ & $-.54 * *$ & .84 & .06 & .77 \\
\hline
\end{tabular}

Note. $N=300$ for all correlations. Reliability coefficients (alphas) are presented along the diagonal. Crisis decision difficulty and confidence measures are standardized.

${ }^{*} p<.05 ; * * p<.01$ 
Table 6

Study 3: Results of Regression Tests of Individual Difference Moderators of C-LEAD on Decision-Making Variables

Crisis Decision Difficulty

Learning Goal Orientation (LGO)

$\begin{array}{lc}\text { C-LEAD } & -.10 \\ \text { LGO } & .07 \\ \text { C-LEAD x LGO } & -.21 * *\end{array}$

Intelligence

\section{C-LEAD}

Intelligence

C-LEAD x Intelligence

Divergent Thinking (DT)

C-LEAD
DT
C-LEAD x DT

C-LEAD

$.21^{* *}$

$-.08$

$-.08$

.05

$-.22 * *$
Crisis Decision Confidence

.07

$-.04$

$29 * *$

$-.17$

$-.34 * *$

$22 * *$

$-.02$

$.15^{*}$

$-.21$

$18^{* *}$

Note. Stepwise entry method used; variables entered in order shown. $N=300$.

${ }^{*} p<.05 ; * * p<.01$ 